\title{
Reizdarm: Reisesouvenir aus den Tropen?
}

\author{
Für eine prospektive Studie wurden in Hamburg Tropenreisende nach ihrer Rückkehr zum \\ neuen Auftreten eines Reizdarmsyndroms befragt. Es ergab sich eine Inzidenz von $>7 \%$.
}

\begin{abstract}
Zwischen Januar 2011 und September 2012 registrierte das Hamburger Institut für Tropenmedizin für eine prospektive Kohortenstudie alle Personen, die sich vor einer Tropenreise impfen ließen. Im Warteraum wurden sie zu gastrointestinalen Symptomen entsprechend den Rom-III-Kriterien befragt. Eine Woche sowie sieben Monate nach der Reise sollten die Teilnehmer dann am Computer einen Fragebogen ausfüllen und per E-Mail zurückschicken.

Von 1.964 registrierten Reisenden antworteten 1.464 direkt nach Rückkehr, 1.190 füllten den Fragebogen auch sieben Monate später aus. Das mittlere Alter lag bei 39,9 \pm 15,7 Jahren, der Frauenanteil bei $53 \%$. Die durchschnittliche Reisedauer betrug 40,8 Tage. 43,3\% der Teilnehmer litten während der Reise min-
\end{abstract}

destens einmal unter mäßigen Symptomen einer Diarrhö.

Die Inzidenz eines neu aufgetretenen Reizdarmsyndroms (RDS) nach den Rom-III-Kriterien betrug sieben Monate nach der Reise 7,2\%. Eine multivariate Analyse ergab, dass biologische Faktoren wie weibliches Geschlecht, vorbestehende Diarrhö-Neigung und eine gastrointestinale Infektion während der Reise das Risiko für das Auftreten eines RDS jeweils verdoppelten. Unter den psychologischen Faktoren spielte Ängstlichkeit gegenüber körperlichen Symptomen die größte Rolle, während Neurotizismus oder Depression keinen Beitrag zum prädiktiven Wert leisteten.

- Löwe B et al. The development of irritable bowel syndrome: a prospective community-based cohort study. Am J Gastroenterol. 2016;111:1320-9

\begin{abstract}
KOMMENTAR
Das Studiendesign schließt Aussagen über die biologischen Pathomechanismen aus. Allerdings stützt der Einfluss einer durchgemachten gastrointestinalen Infektion die Hypothese, dass dem RDS Veränderungen der Immunantwort der Mukosa, des Darmmikrobioms oder des enterischen Nervensystems zugrunde liegen könnten.

Bemerkenswert ist die RDS-Inzidenz von 7\% in der Studie. Tropenreisen an sich müssen offenbar als wesentlicher Risikofaktor für diese Erkrankung angesehen werden. Als Botschaft sollte man sich in jedem Fall behalten: Unabhängig von allen anderen Risikofaktoren wird das Risiko für ein RDS durch gastrointestinale Infektionen verdoppelt.
\end{abstract}

Prof. Dr. med. H. S. FüeßI

\section{Und noch ein fieses Mitbringsel}

Eine 46-jährige Frau litt seit zehn Tagen an Schmerzen und Schwellungen des rechten Arms. Sie war vor kurzem aus der Elfenbeinküste zurück nach England gekommen. An der Streckseite des rechten Unterarms fanden sich mehrere Ulzerationen mit umgebendem Erythem. Unter der Verdachtsdiagnose einer Dermatitis nach Insektenstichen wurde sie antibiotisch behandelt. Am Tag darauf schien sich der Hautbefund verschlimmert zu haben und man bemerkte in den Ulzera Larven, die sich lebhaft bewegten (Abb. A). Vier von innen wurden chirurgisch entfernt (Abb. B, C). Die parasitologische Untersuchung ergab, dass es sich um Larven der Tumbufliege (Cordylobia anthropophaga) handelte. Die Patientin erholte sich danach schnell.

Die auch Mangofliege genannte Schmeißfliegen-Spezies ist im tropischen Afrika endemisch. Die weibliche Fliege legt Eier in warme Kleidung oder sandigen Boden. Nach kurzem Hautkontakt bohrt sich die Larve in das subkutane Gewebe, typischerweise am Rücken, den Nates oder der Rückseite der Extremitäten. Im frühen Stadium werden die Schwellungen meist für Insektenstiche gehalten, da man die Larven nicht erkennt. Die Maden atmen durch eine Öffnung in der Mitte der Schwellung. Streicht man Vaseline oder
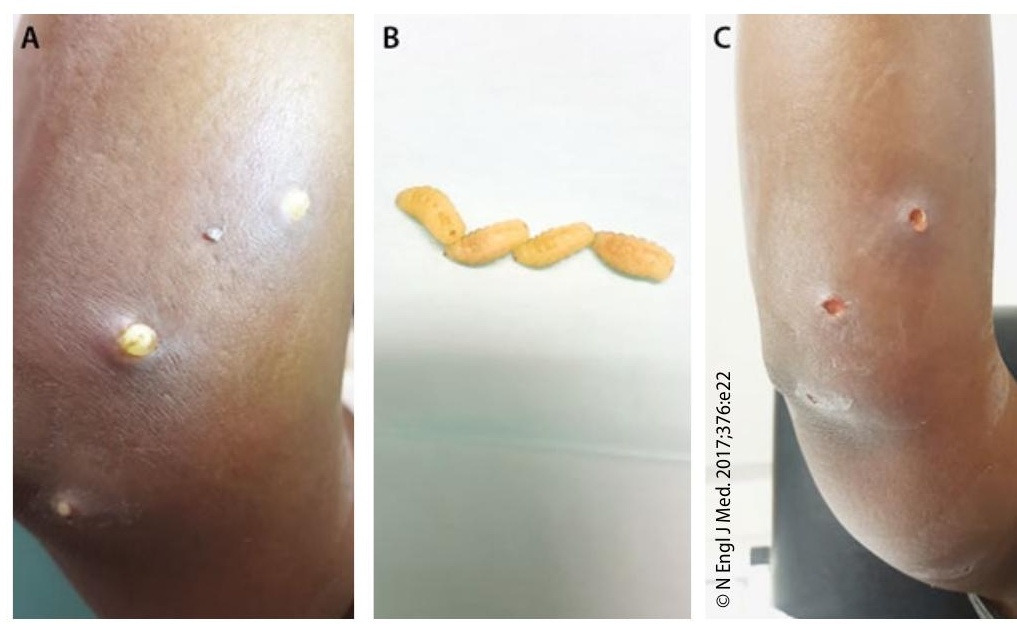

A: Maden im subkutanen Gewebe. B: Extrahierte Exemplare. C: Zustand nach Extraktion.

Öl darauf, kommen die Maden zum Luftholen an die Oberfläche, wo man sie leicht entfernen kann. Manchmal ist aber eine chirurgische Extraktion nötig.

Prof. Dr. med. H. S. FüeßI

- Park JJ, Costello J. Tumbu fly larvae. N Engl J Med. 2017;376:e22 